\title{
Transatlantica
}

Revue d'études américaines. American Studies Journal

$1 \mid 2012$

Le roman policier, littérature transatlantique / Maisons

Hantées

\section{Paule Lévy, dir., Autour De Saul Bellow, Angers, Presses de l'Université d'Angers, 2010}

\section{Élodie Chazalon}

\section{(2) OpenEdition}

\section{Journals}

Édition électronique

URL : https://journals.openedition.org/transatlantica/5704

DOI : $10.4000 /$ transatlantica.5704

ISSN : 1765-2766

Éditeur

Association française d'Etudes Américaines (AFEA)

Référence électronique

Élodie Chazalon, «Paule Lévy, dir., Autour De Saul Bellow, Angers, Presses de l'Université d'Angers,

2010 », Transatlantica [En ligne], 1 | 2012, mis en ligne le 06 janvier 2013, consulté le 31 janvier 2023.

URL : http://journals.openedition.org/transatlantica/5704 ; DOI : https://doi.org/10.4000/

transatlantica. 5704

Ce document a été généré automatiquement le 31 janvier 2023.

\section{(c) (i) $\odot$}

Creative Commons - Attribution - Pas d'Utilisation Commerciale - Pas de Modification 4.0 International - CC BY-NC-ND 4.0

https://creativecommons.org/licenses/by-nc-nd/4.0/ 


\title{
Paule Lévy, dir., Autour De Saul Bellow, Angers, Presses de l'Université d'Angers, 2010
}

\author{
Élodie Chazalon
}

1 Dirigé par Paule Lévy, Autour de Saul Bellow est un ouvrage collectif qui se propose d'analyser les composantes de l'écriture et de l'œuvre de Saul Bellow, y compris ses novellas, fictions brèves moins connues du grand public. Spécialiste de l'écriture juive américaine, auteure de deux monographies et de plusieurs articles sur l'écrivain, à qui elle consacra également sa thèse de doctorat, Paule Lévy donne le ton dès l'introduction.

2 Après quelques éléments biographiques et bibliographiques concernant l'auteur, qui compte parmi les plus grands écrivains américains du vingtième siècle, Paule Lévy souligne la diversité de l'écriture bellovienne, une écriture d'« expérimentation » (5) marquée par le plurilinguisme, l'intertextualité et par un certain anticonformisme. Elle ajoute que les hybridités d'ordre générique, formel, stylistique et linguistique servent de miroir à l'Amérique pluriethnique, urbaine et protéiforme de l'après Seconde Guerre mondiale, et qu'elles sont au centre d'une écriture dont l'iconoclasme ne menace en rien «[la] cohérence et [la] continuité » (6), une caractéristique qui s'applique à la structure même de l'ouvrage.

Douze contributions d'universitaires, dont deux en anglais, traitent de l'oeuvre à «multiples facettes» (6) de Saul Bellow. Partiellement chronologique, la progression de l'ouvrage est la suivante: les deux premiers articles parcourent de façon transversale les grandes lignes de son oeuvre en l'éclairant de références critiques, balisant ainsi ses soubassements intellectuels et historiques; les huit suivants sont axés sur un roman particulier, ou sur une, voire deux nouvelles étudiées dans une perspective comparatiste, et dont ils analysent un aspect spécifique; dans la lignée des deux articles liminaires, les deux derniers articles redirigent le lecteur vers une analyse plus compréhensive, qui sert également de conclusion ouverte. Nonobstant, toutes les contributions s'articulent de façon plus ou moins directe autour de la notion 
d'ambiguïté, et aucune n'omet de relier le style à certains éléments de la vie de l'auteur, ni de replacer l'œuvre dans le contexte socioculturel de l'Amérique de son époque, de sorte que le lecteur, qu'il soit spécialiste ou novice, peut aisément saisir ce qui constitue l'essence de son écriture.

4 Parmi les contributions, il faut noter en premier lieu "Saul Below, un écrivain 'antimoderne'?", dans laquelle Virginia Ricard analyse, principalement au travers de trois romans, le caractère contradictoire de l'œuvre bellovienne qui oscille, jusque dans ses personnages, « entre les opinions de l'écrivain et sa capacité à saisir la modernité » (8). Timea Lönhardt s'inscrit dans la même veine dans «Entre misogynie et peur du féminisme: l'anti-radicalisme de Saul Bellow». Elle s'appuie sur les romans publiés après 1970 pour nuancer la lecture antiféministe des œuvres de Saul Bellow, une misogynie qu'elle impute à «un refus de l'endoctrinement» (26) plus qu'à la gynophobie.

5 Dans les huit contributions qui suivent, la notion d'ambiguïté est traitée de façon polysémique. Dans leurs travaux respectifs, Gwen Le Cor et Jean-Yves Pellegrin mettent en lumière la dichotomie dévoilement/dissimulation en répertoriant quelques-uns des procédés rhétoriques et narratifs (multiples focalisations, tropes de la distorsion, etc.) qui contribuent aux « effets de brouillage » (29). De plus, ils analysent la mise en scène du sentiment anomique des «dangling men » belloviens via le thème du masque. Les autres contributeurs s'intéressent au motif de la répétition aliénante et mortifère: obsession du dédoublement rendue par les distorsions spéculaires dans Herzog (Paule Lévy), délocalisations, corps souffrants, et diffractions de l'imaginaire juif via l'esthétique grotesque dans «Leaving the Yellow House (Marie Liénard-Yeterian), " répétition du motif vénal » (83) et «liquidation » de l'héritage juif dans "A Silver Dish» (Géraldine Chouard), ubiquité de la corruption et musiques lancinantes dans "Cousins» et «Him With His Foot in His Mouth» (Jennifer Kilgore), télescopages philosophiques, mythologiques et avatars de la mémoire dans The Bellarosa Connection (Emmanuel Vernadakis), et enfin digressions et répétitions de l'« écriture du décalage " dans Ravelstein (Régine Camps-Robertson). Les deux articles qui clôturent l'ouvrage replacent l'œuvre dans son contexte socio-historique et ses fondements philosophiques. Dans «De Saul Bellow à Walker Percy: Portrait d'une Amérique en crise ", Gérald Préher mène une étude comparative des «mondes fictionnels de Bellow et Percy » (132), en y soulignant l'étroite corrélation entre urbanité et exotisme, et entre l'histoire des protagonistes et l'Histoire d'une Amérique contemporaine dépeinte sur le mode cataclysmique. Elyette Labarthe passe enfin en revue l'œuvre de Saul Bellow et rend hommage à son éclectisme et à son caractère paradoxal. «La querelle bellovienne autour de l'humanisme " explore ainsi la démarche intellectuelle, et sans cesse évolutive, d'un auteur qui, soucieux de «briser les cadres de la connaissance intellectuelle » (144), s'ancre profondément dans le postmodernisme.

6 Le mérite de cet ouvrage réside dans le fait qu'il traite également des fictions brèves, qui sont d'ordinaire des laissés-pour-compte. À l'instar de l'œuvre de Saul Bellow, ce livre est à la fois un microscope placé sur la société américaine et sur l'héritage culturel de l'auteur, et un télescope donnant à voir l'interaction entre l'histoire des idées et l'Histoire de l'humanité. Grâce à cet ouvrage à la fois centrifuge et centripète, le lecteur voyage en effet « autour », mais aussi, et peut-être surtout, au cœur de l'œuvre de Saul Bellow. 
INDEX

Thèmes : Recensions

\section{AUTEUR}

ÉLODIE CHAZALON

Université de Perpignan-Via Domitia 